\title{
Thermal shielding of an emerging active region ${ }^{\star}$
}

\author{
S. Régnier \\ Jeremiah Horrocks Institute, University of Central Lancashire, Preston, Lancashire, PR1 2HE, UK \\ e-mail: SRegnier@uclan.ac.uk \\ Received 17 August 2011 / Accepted 17 July 2012 \\ ABSTRACT \\ Context. The interaction between emerging active regions and the pre-existing coronal magnetic field is important for better under- \\ standing the mechanisms of storage and release of magnetic energy from the convection zone to the high corona. \\ Aims. We describe the first steps of an emerging active region within a pre-existing quiet-Sun corona in terms of the thermal and \\ magnetic structure. \\ Methods. We used unprecedented spatial, temporal and spectral coverage from the Atmospheric Imager Assembly (AIA) and from \\ the Helioseismic and Magnetic Imager (HMI) on board the Solar Dynamics Observatory (SDO). \\ Results. Starting on 30 May 2010 at 17:00 UT, we followed the emerging active region AR11076 within a quiet-Sun region for $8 \mathrm{~h}$. \\ Using several SDO/AIA filters that cover temperatures from $50000 \mathrm{~K}$ to $10 \mathrm{MK}$, we show that the emerging process is characterised \\ by a thermal shield at the interface between the emerging flux and pre-existing quiet-Sun corona. \\ Conclusions. The active region 11076 is a peculiar example of an emerging active region because (i) the polarities emerge in a \\ photospheric quiet-Sun region near a supergranular-like distribution, and (ii) the polarities that form the bipolar emerging structure \\ do not rotate with respect to each other, which indicates a slight twist in the emerging flux bundle. There is a thermal shield at the \\ interface between the emerging active region and the pre-existing quiet-Sun region. The thermal shielding structure deduced from all \\ SDO/AIA channels is strongly asymmetric between the two polarities of the active region, suggesting that the heating mechanism for \\ one polarity is probably magnetic reconnection, whilst it is caused by increasing magnetic pressure for the opposite polarity.
}

Key words. Sun: corona - Sun: evolution - Sun: UV radiation - Sun: surface magnetism

\section{Introduction}

Flux emergence is a process that transports magnetic energy and plasma from the convection zone into the solar atmosphere. The standard models of flux emergence (see reviews by Archontis 2008; Hood et al. 2011) are based (i) on the buoyancy of twisted flux tubes that are generated near the tachocline by the global dynamo action, or (ii) on the generation of a small-scale magnetic field in flux sheet regions in a thin layer near the photosphere. Because the magnetic flux is transported by convective motions, emerging regions are most likely to appear at the centre of supergranular cells where strong radial upflows and/or horizontal flows are observed (Leighton et al. 1962). The emerging flux is first observed in the photosphere as newly formed, highly concentrated magnetic elements. The magnetic elements are then growing in size and moving apart to build magnetic polarities such as pores and sunspots. Important features of emerging twisted flux tubes are an increase of the total unsigned magnetic flux, the separation of polarities for increasing flux, and the rotation of magnetic polarities with respect to each other due to the transport of twist (or helicity) into the corona (Zwaan 1985; López Fuentes et al. 2003). Extensive observations of emerging active regions in quiet-Sun areas or in already emerged active regions have been reported previously (e.g., Zwaan 1985; van Driel-Gesztelyi et al. 2000; Okamoto et al. 2008; Pariat et al. 2009; Canou et al. 2009; Harra et al. 2010; Gömöry et al. 2010; Vargas Domínguez et al. 2011). Still, two parts of the flux emergence into the corona have not yet been tackled in detail


http://www. aanda.org
}

due to the lack of high cadence and wide temperature coverage in terms of magnetic field and EUV emission: (i) the very first steps of the emergence (the first few hours) before pores are formed and the emerged flux has just reached coronal heights, and (ii) the steady and impulsive response of the corona during that period.

AR 11076 was observed to emerge on 30 May 2010 in the southern hemisphere. To determine the initial time of the flux emergence, we used the capabilities of SDO/HMI by combining line-of-sight magnetic field and continuum images to pinpoint the first signature of the emergence. Hence we define 30 May 2010 at 17:00 UT as the start of the emergence. We combined SDO/HMI and SDO/AIA data to study the interaction of the emerging magnetic field and the pre-existing quietSun corona. We restricted the study to the first eight hours of emergence just before the formation of a pore. The sunspots (umbra and penumbra) were formed later on 31 May around 12:00 UT. We also give a physical interpretation of a thermal shield formation.

\section{Thermal and magnetic evolution from SDO/AIA and SDO/HMI data}

We describe the evolution of the emerging active region 11076 during eight hours between 17:00 UT on 30 May 2010 to 01:00 UT on 31 May 2010. We used the SDO/HMI (Scherrer et al. 2012) and SDO/AIA instruments to study the magnetic and thermal structure of the emerging region. SDO/AIA data are level-1 images using a first approximation of the calibration, which does not change the results reported in this Letter. 
The pixel size is $0.56^{\prime \prime}$ and the time cadence for this study is $45 \mathrm{~s}$ for SDO/HMI and $36 \mathrm{~s}$ for SDO/AIA. SDO/AIA capabilities and thermal responses are described in Lemen et al. (2012; see also O'Dwyer et al. 2010). We accordingly define the temperature of a channel as the temperature of the peak of emission as reported by Lemen et al. (2012), keeping in mind that all broad-band channels are multithermal.

In the top row of Fig. 1, we display the evolution of the photospheric SDO/HMI line-of-sight magnetic field at three different times (17:00 UT, 21:00 UT, and 01:00 UT). The quiet-Sun magnetic-field distribution at 17:00 UT looks like a supergranule magnetic field (large-scale convection cell), and corresponds to the pre-existing coronal magnetic configuration. In the top left of Fig. 1, the supergranular boundaries with the highest magneticfield strength are highlighted with ellipses. The supergranularlike magnetic field is not unipolar but composed of mixed positive and negative polarities. The bipolar structure of AR 11076 appears clearly in the next two frames with increasing magnetic field strength and area. In the middle top row of Fig. 1, we annotate the different polarities that are important for the evolution: $\mathrm{N} 1, \mathrm{P} 1$ and $\mathrm{P} 3$ denote the negative $(\mathrm{N})$ and positive $(\mathrm{P})$ polarities of the pre-existing quiet-Sun magnetic field, and N2 and P2 are the polarities associated with the emerging active region. We note that during the first eight hours of the emergence, the direction between $\mathrm{N} 2$ and $\mathrm{P} 2$ polarities remained the same along the east-west direction. To complement the evolution of the photospheric magnetic field, we plot in Fig. 2 the total unsigned magnetic flux (black curve), and the positive (in red) and negative (in blue) magnetic fluxes. The magnetic fluxes are computed from the line-of-sight component of the magnetic field transformed into a vertical component. Obviously, the total unsigned flux is increased, as it should for an emerging active region, but the negative flux $(+18 \%)$ contributes mainly to this increase, whilst the positive flux increases at a slower rate $(+8 \%)$. This gives an emergence rate for the whole area of $4 \times 10^{19} \mathrm{Mx} \mathrm{h}^{-1}$ with $2.7 \times 10^{19} \mathrm{Mx} \mathrm{h}^{-1}$ for the negative flux and $1.2 \times 10^{19} \mathrm{Mx} \mathrm{h}^{-1}$ for the positive flux. The emergence process is not supposed to change the net magnetic flux, but the observed magnetic flux does not take into account the geometry or inclination of the magnetic field (in other words, the transverse components of the magnetic field). The eight hours of this time series are long enough to produce a substantial amount of magnetic flux through the photosphere, which forms magnetic flux concentrations consistent with the recent numerical simulations of emerging active regions by Cheung et al. (2010). However, according to these authors, pores and sunspots are forming later during the emergence phase. Our observations are supporting the results obtained by Cheung et al. (2010).

In rows 2-6 of Fig. 1, the EUV emission lines show the behaviour of the emerging active region from $50000 \mathrm{~K}$ to several million degrees. The intensity maps of the $304 \AA$ and $171 \AA$ channels show much fine structuring within the emerging flux, including dark material, which is presumably cool material. The emission for the other three channels is more diffuse (and near the noise level for the $94 \AA$ channel). From the time series (Fig. 1), it is clear that hot channels such as $193 \AA, 211 \AA$, and $94 \AA$ show the large-scale magnetic field lines of the emerging flux bundles and thus interface more with the pre-existing magnetic field. We determined the expansion of the emerging flux in the north-south and east-west directions by measuring the full-width at half-maximum of the intensity variation at a given time. As expected, the expansion of the emerged magnetic field is larger in regions of weak magnetic field strength (north-south
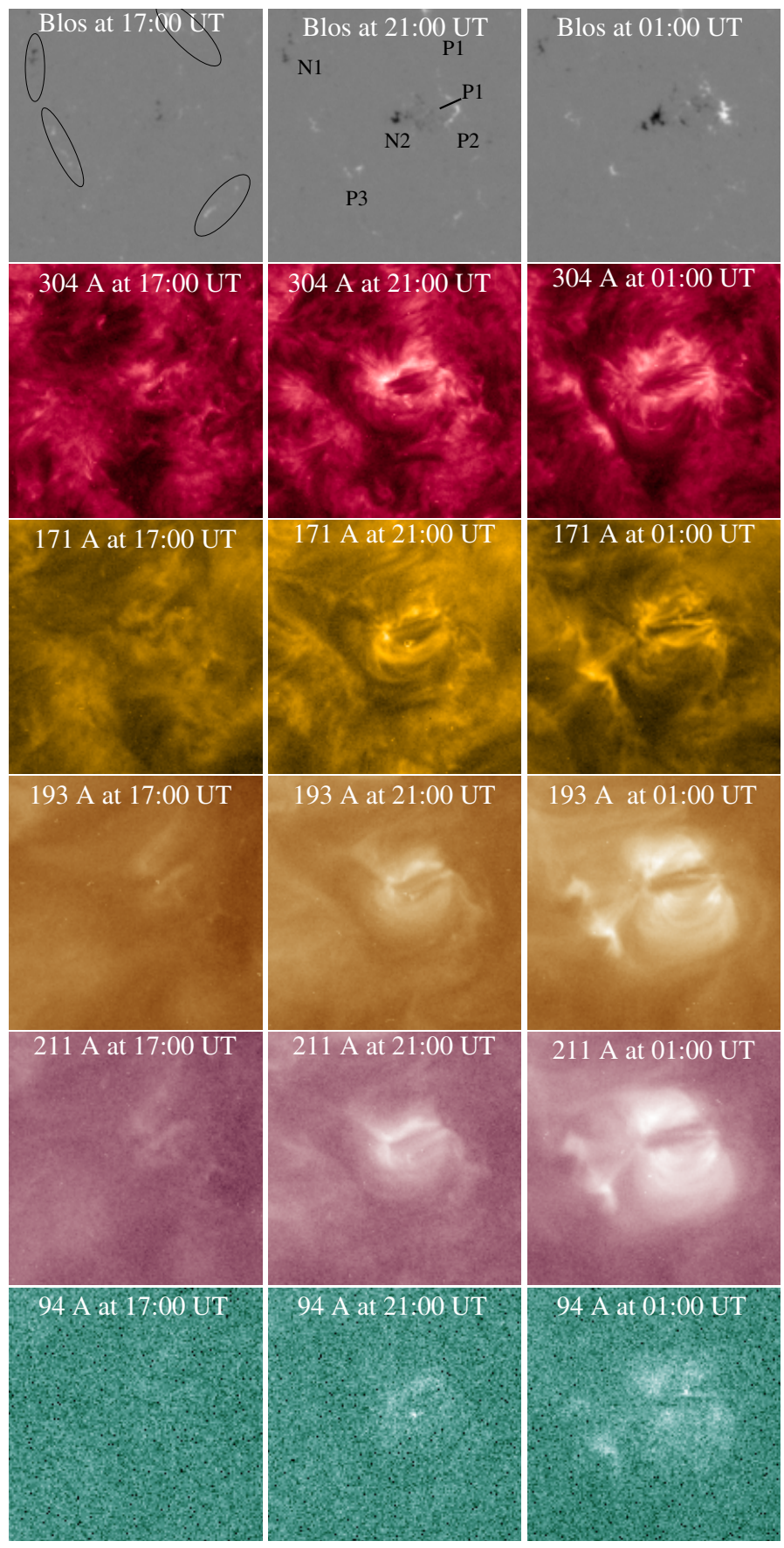

Fig. 1. Time series of images showing the emergence of the active region at three different times during the eight hours: the line-of-sight magnetic field from SDO/HMI (top row) scaled between $-800 \mathrm{G}$ and $800 \mathrm{G}$, chromospheric and coronal response from SDO/AIA at $304 \AA$, $171 \AA, 193 \AA, 211 \AA$ and $94 \AA$ (from top to bottom). The field-of-view is $90^{\prime \prime}$ in both directions centred on $\left(-370^{\prime \prime},-305^{\prime \prime}\right)$ at 17:00 UT. See text for details. Movies of the time evolution of the emergence are provided as online material.

direction in this case) than along the main axis of the active region (east-west direction):

- the length in the north-south direction is about $18 \mathrm{Mm}$ at 21:00 UT and 28 Mm at 01:00 UT. The rate of expansion in the north-south direction can then be estimated at $2.5 \mathrm{Mm} \mathrm{h}^{-1}$. This rate of expansion will give a typical active region of size $120 \mathrm{Mm}$ after two days;

- there is no measurable expansion in the east-west direction. 


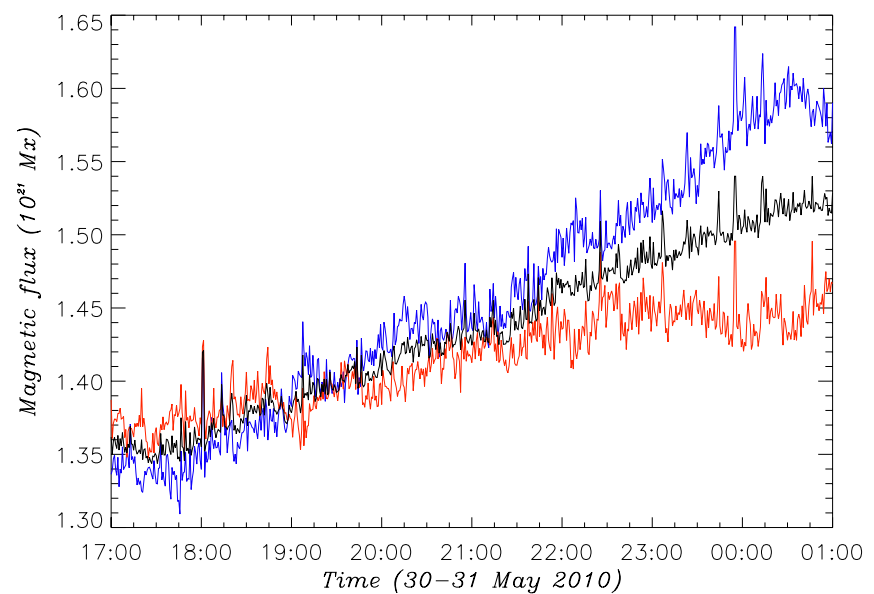

Fig. 2. Total magnetic flux evolution over the area of Fig. 1 during eight hours: the unsigned flux is depicted in black (divided by a factor of two), the negative flux in blue (in absolute value), and the positive flux in red. The magnetic flux is given in units of $10^{21} \mathrm{Mx}$.

The EUV brightenings in the south-east part of the active region (coordinates $X=-320^{\prime \prime}, Y=-320^{\prime \prime}$ in Fig. 1 rows 4 and 5 at 01:00 UT) show the interaction between the pre-existing coronal field and the emerging flux, which creates a magnetic connection between the two polarities (P3 and N2) as shown by the EUV loops.

To investigate the location and process of the heating, we plot the intensity variation for five SDO/AIA channels averaged over three hours (from 22:00 to 01:00 UT). In the top of Fig. 3, the intensity variations in the east-west direction along the axis between the negative and positive polarities of the emerging region are plotted. All five curves have basically the same shape with a clear double peak structure where the edges of the emerging flux region are located. The peaks are not symmetric: the peak near the negative polarity (left-hand side) is at least $20 \%$ higher than the peak associated with the positive polarity (righthand side). This asymmetry is important for determining the processes responsible for heating the emerging configuration. Because the magnetic field strength is similar in both polarities (about $800 \mathrm{G}$ ), the asymmetry indicates two different processes: (i) magnetic reconnection of magnetic field lines of different orientations that converts the magnetic energy into kinetic and thermal energy, and (ii) magnetic compression due to the increase of magnetic pressure when the geometry of the field does not allow magnetic reconnection to occur. In Fig. 4, we draw a 2D sketch that summarises the processes at play during the emergence of the active region, and which produce the asymmetry in the double peak emission. As indicated in the top row of Fig. 1, N2 and P2 form the emerging bipolar region corresponding to the field lines in red. We notice that on the right-hand side, the field lines are anchored in the positive polarities (P1 and P2) with the same orientation, which is more favourable for a heating mechanism such as the magnetic compression. On the left-hand side of the emerging flux, the magnetic topology is more complex and it is more likely to produce magnetic reconnection. The highest peak being on the left-hand side is explained by the impulsive nature of the magnetic reconnection compared to the slow process of magnetic compression. In the movie that shows the evolution in the $171 \AA$ channel, we observe that magnetic field lines originating in $\mathrm{N} 2$ are reconnected from $\mathrm{P} 2$ to $\mathrm{P} 3$.

In the bottom of Fig. 3, we plot the intensity variation in the south-north direction perpendicular to the axis of the active region. We find again the double peak structure, but the
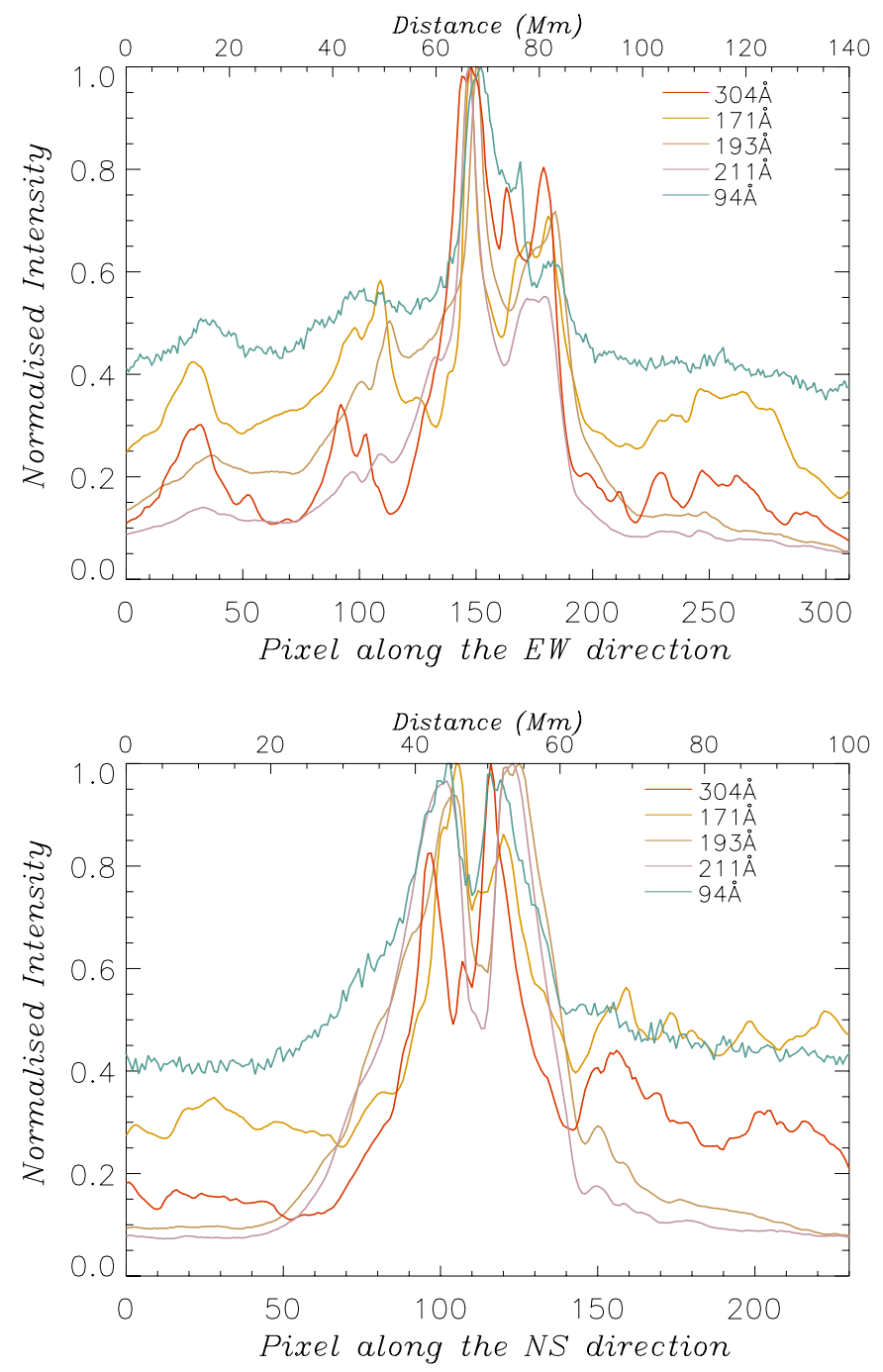

Fig. 3. Intensity variation averaged over three hours (22:00 to 01:00 UT) for all five SDO/AIA channels: along the east-west direction at the NS location of $-300^{\prime \prime}$ (top), and along the south-north direction at the initial EW location of $-375^{\prime \prime}$ (bottom). The intensity is normalised to the maximum intensity for the sake of comparison.

intensity variations are symmetric: this probably indicates a heating mechanism such as magnetic compression. A common feature to both intensity variation plots is the double-peak structure: two peaks on the side that can be explained by either magnetic reconnection or magnetic compression, and an emission dip. The dip is caused by emerging cool plasma that appears as dark material in the EUV images of Fig. 1. This is also a common feature in models of magnetic flux emergence (Magara 2001; Archontis et al. 2007; Martínez-Sykora et al. 2008) in which the emerging flux rope has cool material at its centre. These observations of a newly emerged flux show similar timescale and spatial-scale as the simulation of Cheung et al. (2010), only the magnetic field strength is noticeably different $(800 \mathrm{G}$ in the observation, $3000 \mathrm{G}$ in the simulation).

\section{Discussion and conclusions}

We observed the first eight hours of an emerging active region. The peculiarities of this emerging flux are (i) that the new polarities emerge near a supergranular-like boundary; and (ii) that no rotation of the polarities that form the emerging active region 


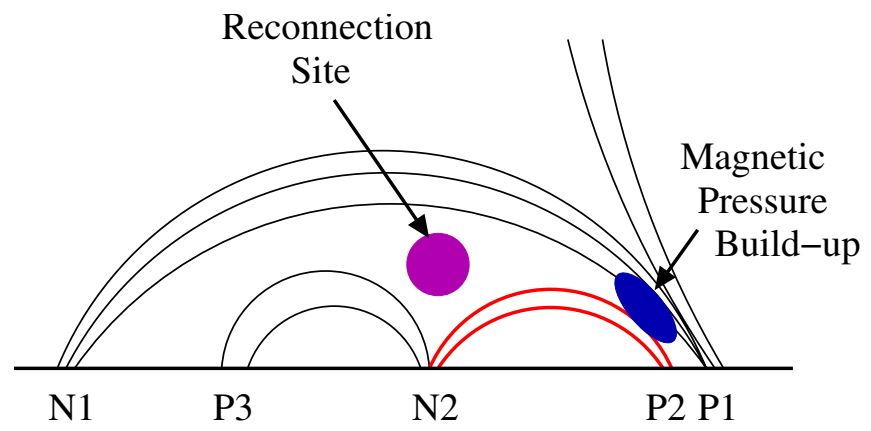

Fig. 4. Sketch of the magnetic field structure of the emerging active region as deduced from the distribution of polarities in the middle top row of Fig. 1. The field lines in red characterise the emerging magnetic field. East is on the left-hand side.

with respect to each other is observed. This strongly suggests that the emergence process is either due to the buoyancy of a flux tube that contains a slight twist, or that it is associated with a flux sheet near the solar surface.

The SDO/AIA observations provide an unprecedented view of the thermal structure of an emerging active region and its interaction with the hot corona. To analyse the thermal structure of the emerging active region, we plotted the average intensity variation for the different wavelengths for the last three hours of the time series when the size of the active region remained almost constant. We observed the thermal shielding of the emerging flux at the interface with the pre-existing quiet-Sun magnetic field: all wavelength channels exhibit two strong peaks at the interface between the emerging region and the pre-existing corona. By comparing with 3D models (Magara 2001; Archontis et al. 2007; Martínez-Sykora et al. 2008), we deduced that the observed asymmetry in the intensity variation between the east and west sides is due to two different processes that are depicted in Fig. 4:

(i) On the west side, the build-up of magnetic pressure in a system of magnetic field lines with the same orientation is responsible for the increase of temperature at the shield. (ii) On the east side, magnetic reconnection can be invoked to heat the shield owing to the opposite orientation of field lines and the complex geometry of the field.

We were able to characterise the thermal structure of an emerging active region in the quiet Sun. In addition, the magnetic flux emergence process also generates impulsive events such as external magnetic reconnection between the emerging and pre-existing magnetic fields, and internal magnetic reconnection within the emerging flux bundles. These processes and the way they contribute to the emergence will be discussed in a forthcoming paper.

Acknowledgements. I thank the referee for her/his comments, which have improved the manuscript. I would like to thank Vasilis Archontis for fruitful discussions on flux emergence. The SDO data have been collected from the University of Central Lancashire database. The data used are provided courtesy of NASA/SDO and the AIA and HMI science teams.

\section{References}

Archontis, V. 2008, J. Geophys. Res. (Space Phys.), 113, A03S04 Archontis, V., Hood, A. W., \& Brady, C. 2007, A\&A, 466, 367

Canou, A., Amari, T., Bommier, V., et al. 2009, ApJ, 693, L27

Cheung, M. C. M., Rempel, M., Title, A. M., \& Schüssler, M. 2010, ApJ, 720, 233

Gömöry, P., Beck, C., Balthasar, H., et al. 2010, A\&A, 511, A14

Harra, L. K., Magara, T., Hara, H., et al. 2010, Sol. Phys., 263, 105

Hood, A. W., Archontis, V., \& MacTaggart, D. 2011, Sol. Phys., 74

Leighton, R. B., Noyes, R. W., \& Simon, G. W. 1962, ApJ, 135, 474

Lemen, J. R., Title, A. M., Akin, D. J., et al. 2012, Sol. Phys., 275, 17

López Fuentes, M. C., Démoulin, P., Mandrini, C. H., Pevtsov, A. A., \& van Driel-Gesztelyi, L. 2003, A\&A, 397, 305

Magara, T. 2001, ApJ, 549, 608

Martínez-Sykora, J., Hansteen, V., \& Carlsson, M. 2008, ApJ, 679, 871

O’Dwyer, B., Del Zanna, G., Mason, H. E., Weber, M. A., \& Tripathi, D. 2010, A\&A, 521, A21

Okamoto, T. J., Tsuneta, S., Lites, B. W., et al. 2008, ApJ, 673, L215

Pariat, E., Masson, S., \& Aulanier, G. 2009, ApJ, 701, 1911

Scherrer, P. H., Schou, J., Bush, R. I., et al. 2012, Sol. Phys., 275, 207

van Driel-Gesztelyi, L., Malherbe, J.-M., \& Démoulin, P. 2000, A\&A, 364, 845

Vargas Domínguez, S., MacTaggart, D., Green, L., van Driel-Gesztelyi, L., \& Hood, A. W. 2011, Sol. Phys., 178

Zwaan, C. 1985, Sol. Phys., 100, 397 


\section{Appendix A: Time evolution of the emerging active region}

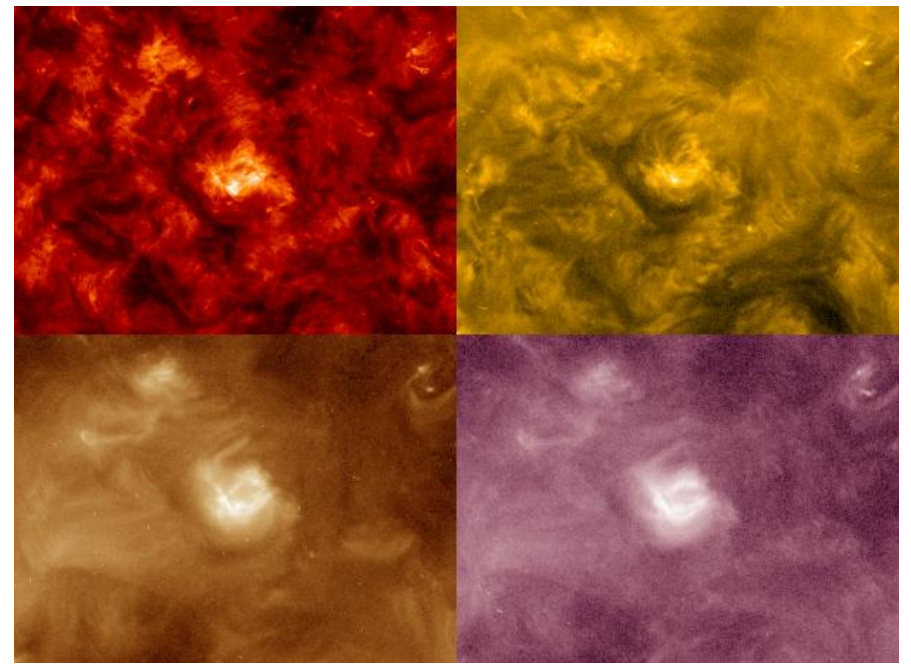

Fig. A.1. Still image of the SDO/AIA movie at 21:30 UT on 30 May 2010: $304 \AA$ (top left), $171 \AA$ (top right), $193 \AA$ (bottom left), $211 \AA$ (bottom right). The field-of-view is $180^{\prime \prime} \times 132^{\prime \prime}$.

A movie of the evolution of the emerging active region during eight hours (17:00 UT on 30 May 2010 to 01:00 UT on 31 May 2010, 36 s time cadence) is supplied as online material.

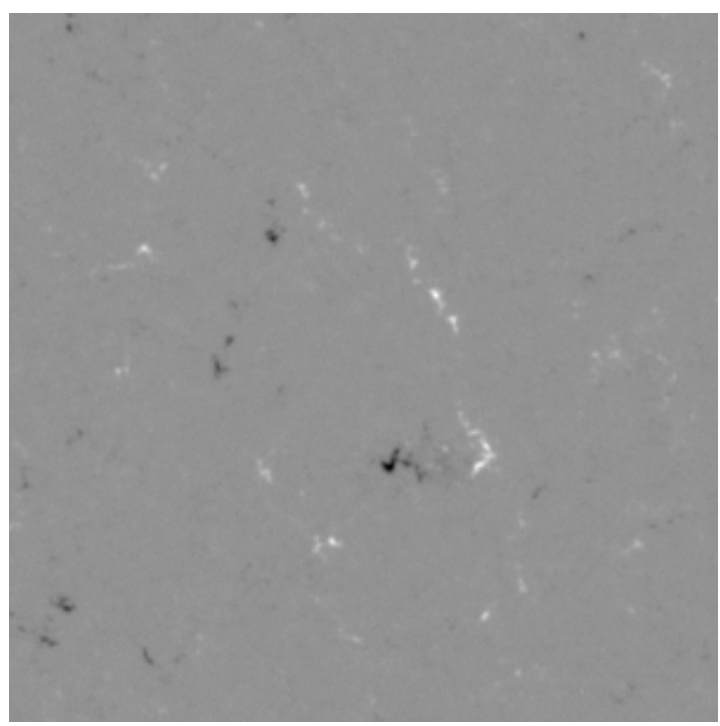

Fig. A.2. Still image of the SDO/HMI line-of-sight magnetic field at $21: 30$ UT on 30 May 2010. The field-of-view is $180^{\prime \prime} \times 180^{\prime \prime}$ centred at $\left(-320^{\prime \prime},-280^{\prime \prime}\right)$ at this particular time (reference frame for the cross-correlation).

The movie of the evolution of the magnetic field is also provided using a larger field-of-view to clearly see the supergranule pattern. The movie is for the same period of eight hours and with a cadence of $45 \mathrm{~s}$. 